BBA 52609

\title{
Parinaric acid as a sensitive fluorescent probe for the determination of lipid peroxidation
}

\author{
Frans A. Kuypers ${ }^{a}$, Jeroen J.M. van den Berg ${ }^{b}$, Casper Schalkwijk ${ }^{b}$, \\ Ben Roelofsen ${ }^{b}$ and Jos A.F. Op den Kamp ${ }^{b}$ \\ ${ }^{a}$ Children's Hospital Oakland Research Institute, Oakland, CA (U.S.A.) and ${ }^{b}$ Department of Biochemistry, \\ State University of Utrecht, Utrecht (The Netherlands)
}

(Received 25 February 1987)

Key words: Lipid peroxidation; Parinaric acid; Fluorescent probe; Phosphatidylcholine; (Bovine liver)

The decrease in fluorescence of conjugated polyenic acyl chains is used as a sensitive assay for lipid peroxidation. The fatty acid cis-trans-trans-cis-9,11,13,15-octadecatetraenoic acid (cis-parinaric acid) is introduced into liposomal membranes as free fatty acid or, by using the PC specific transfer protein from bovine liver, as 1-palmitoyl-2-cis-parinaroyl-sn-glycero-3-phosphocholine. The peroxidation process as monitored by the decrease in fluorescence intensity is compared with other peroxidation assay systems. Applications of the new assay system are discussed.

\section{Introduction}

The oxidation of lipids in biological systems via uncontrolled free radical chain reactions has profound consequences and is a feature of many types of cell injury [1]. Especially the polyunsaturated fatty acids, like arachidonic acid or docosahexaenoic acid, are very sensitive to this process and can undergo peroxidation in their free carboxylate form as well as esterified in phospholipids. Peroxidation of the acyl chains of lipids results in the formation of a great variety of products, ultimately in the breakdown of the carbonyl chain to form compounds like alkanes and malondialdehyde. In a membrane, this can cause direct effects by changes in the membrane

Abbreviations: PC, phosphatidylcholine; POPC, 1-palmitoyl2-oleoylPC; DLPC, 1,2-dilinoleoylPC.

Correspondence: J.J.M. van den Berg, Laboratory of Biochemistry, State University of Utrecht, Padualaan 8, NL-3584 Utrecht, The Netherlands. structure as a result of changes in the lipid core, or indirect effects as a result of the reaction of peroxidation products with other membrane constituents [2]. A great variety of reactive free radicals, originating from several sources, can interact with neighbouring components in the cell. A very efficient complex system of protective mechanisms is normally present in a cell to minimize the deleterious effects these radicals can initiate. Damage will occur only if these mechanisms are overcome. Because the enormous complexity of the processes involved, the chemical mechanisms are still not fully understood [3] and require further investigation.

The significance of lipid peroxidation has led to the development of several methods to monitor this process, which have recently been revicwed [4]. They involve the determination of (i) various products of peroxidation, (ii) uptake of oxygen, and (iii) loss of lipid substrate, more specifically, the loss of polyunsaturated fatty acyl chains.

The fluorescent polyunsaturated fatty acid, cis-parinaric acid, and its all-trans isomer are 
spectroscopically well defined membrane probes $[5,6]$. They have been used, as free acid or esterified in phospholipid analogues, to study lipid-lipid and lipid-protein interactions in lipid model systems as well as in biological membranes [7,8]. If exogenously administered, the free fatty acid incorporates readily into the membrane [9]. Transfer proteins have made the use of phospholipid analogues more applicable by facilitating their insertion into membrane systems [10,11]. The probe molecules can be expected to cause minimal disturbance of the lipid bilayer, since the polyene chromophore does not differ much from the mono-unsaturated acyl chain that is normally found in a membrane at high concentrations [12], making them almost perfect lipid probes. The probe, however, is - as a result of its polyenic structure - very sensitive towards peroxidation, and in all studies mentioned above, great care has been taken to avoid this phenomenon. We report here the use of the peroxidative degradation of this probe, indicated by a decrease in its fluorescence, as an assay to monitor the sensitivity of a membrane towards oxidative stress. Results are compared with well-established procedures and possible applications are discussed.

\section{Materials and Methods}

1-Palmitoyl-2-oleoylphosphatidylcholine (POPC), 1,2-dilinoleoylphosphatidylcholine (DLPC), egg phosphatidylcholine (egg PC) and egg phosphatidate were purchased from Sigma (St. Louis, MO, U.S.A.), cis-parinaric acid was obtained from Molecular Probes (Junction City, OR, U.S.A.) and 1-palmitoyl-2-cis-parinaroyl phosphatidylcholine was synthesized as described previously [13] and generously donated by colleages of the Biochemistry Department (Utrecht, The Netherlands). ParinaroylPC was also received as a kind gift from Unilever Research Laboratorium (Vlaardingen, The Netherlands). Both probes were stored in ethanol under argon at $-20^{\circ} \mathrm{C}$.

\section{Preparation of phospholipid vesicles}

Lipid mixtures of PC and phosphatidate (100:5, $\mathrm{mol} / \mathrm{mol}$ ), dissolved in chloroform/methanol $(2: 1, v / v)$, were dried under nitrogen and dispersed in a buffer containing $150 \mathrm{mM} \mathrm{NaCl} / 10 \mathrm{mM}$
Tris- $\mathrm{HCl}$ ( $\mathrm{pH}$ 7.4) (referred to as 'buffer' throughout). The buffer was filtered before use through a Millipore filter $(0.47 \mu \mathrm{m})$, degassed and stored under argon. The lipid dispersion in buffer was sonicated under nitrogen at $70 \mathrm{~W}$ for 3-6 min and centrifuged at $100000 \times g$ for $30 \mathrm{~min}$. The recovery of $\mathrm{PC}$ in the vesicle containing supernatant was normally $50-80 \%$.

Donor vesicles composed of the fluorescent parinaroylPC were prepared by injection of a concentrated ethanolic solution into the buffer.

\section{$P C$ transfer protein}

The PC specific transfer protein from bovine liver was purified as described before [14] and stored in $50 \%$ glycerol at $-20^{\circ} \mathrm{C}$. Prior to use, glycerol was removed by overnight dialysis against buffer at $4^{\circ} \mathrm{C}$. The volume of the resulting protein solution was reduced by concentration against flake poly(ethylene glycol) (Aquacide III, Calbiochem, San Diego, CA, U.S.A.).

\section{Fluorescence measurements}

Fluorescence measurements were carried out at $25^{\circ} \mathrm{C}$ in a thermostated cuvet equipped with a stirring device using a Perkin Elmer MPF-3 Fluorescence Spectrophotometer. The excitation wavelength was $324 \mathrm{~nm}$ (slit width $3 \mathrm{~nm}$ ) and the emission wavelength $413 \mathrm{~nm}$ (slit width $11 \mathrm{~nm}$ ).

Without mixing, the fluorescence intensity was unstable, probably due to local bleaching of the probe.

\section{Lipid analysis}

Extraction of lipids was performed as described by Rose and Oklander [15]. The phospholipid content was determined as inorganic phosphate according to Rouser et al. [16]. The fatty acid composition of the extracts was determined by gas-liquid chromatography (GLC) after conversion to their methyl esters according to Morrison and Smith [17].

GLC was performed on a Packard series 7300 gas chromatograph employing glass tubular columns $(2 \mathrm{~m} \times 2 \mathrm{~mm}$ i.d.) packed with $3 \%$ Silar $5 \mathrm{CP}$ on Gas Chrom Q 11 (80-100 mesh - Supelco, Bellefonte) at a column temperature of $180^{\circ} \mathrm{C}$.

Increase in diene conjugation was determined according to Klein [18], measuring a $A_{233} / A_{207}$ 


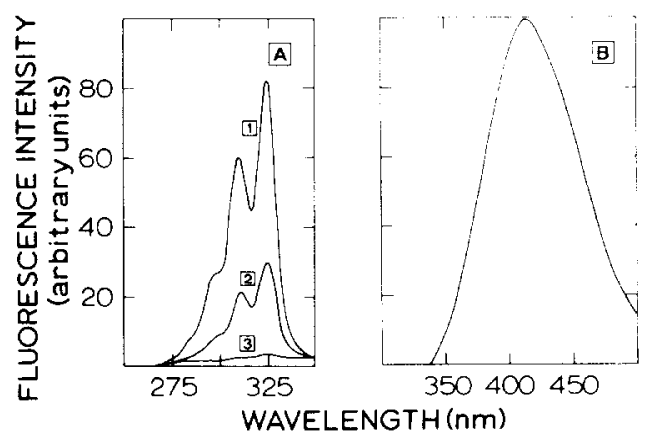

Fig. 1. Fluorescence excitation and emission spectra of cisparinaric acid in egg $\mathrm{PC}$ vesicles at $25^{\circ} \mathrm{C}$. Fluorescence intensity is given in arbitrary units. (A) Excitation spectra of cisparinaric acid in egg $\mathrm{PC}$ vesicles before (1), during (2), and after (3) $\mathrm{H}_{2} \mathrm{O}_{2}$ and $\mathrm{Cu}^{2+}$ induced peroxidation. Fluorescence intensity was measured at an emission wavelength of $413 \mathrm{~nm}$ with slit-width $11 \mathrm{~nm}$, excitation was scanned with slit-width 3 nm. (B) Emission spectrum of cis-parinaric acid in egg PC vesicles. Excitation was at $324 \mathrm{~nm}$ with slit-width $3.5 \mathrm{~nm}$ and emisison was scanned with slit-width $11 \mathrm{~nm}$.

oxidation index in a Unicam SP 1700 double-beam ultraviolet spectrophotometer. The production of thiobarbituric acid-reactive substances was measured according to Bidlack and Tappel [19].

Absorbance values determined at $532 \mathrm{~nm}$ were expressed as equivalents malondialdeliyde, using a standard malondialdehyde solution freshly prepared by hydrolysis with $0.1 \mathrm{M} \mathrm{HCl}$ from 1,1,3,3-tetraethoxypropane [20]. Standards -were treated as samples, giving a molar absorbance coefficient of 84000 .

\section{Results}

The use of cis-parinaric acid

Fig. 1 shows the fluorescence excitation (A) and emission (B) spectra of cis-parinaric acid in egg PC vesicles. The location of the broad emission peak in Fig. 1B is largely independent of the environment of the probe molecule. The fluorescence quantum yield, however, strongly depends on its environment. When small volumes of an ethanolic parinaric acid solution are added to buffer, the fluorescence is strongly quenched due to the probe-probe interactions. If membranes are present in the buffer, the fluorescent fatty acid will be partitioned between the water phase and the membrane. The probe-probe interaction will decrease because of the dilution of the probe in the membrane, resulting in an increase in fluorescence intensity. The equilibrium of incorporation in the membrane is reached within 1-2 min, as is indicated by the rate of increase of fluorescence intensity. In the membrane, the quantum yield of parinaric acid depends on the physical properties of the membrane itself as well as on the concentration of the probe $[5,6]$.

In Fig. 2, the relation is shown between the fluorescence intensity and the concentration of lipid and parinaric acid in an egg PC vesicle suspension. Similar results are found for several membranes, indicating that a molar concentration of up to about $1 \%$ gives a linear relationship between the concentration of the probe and the
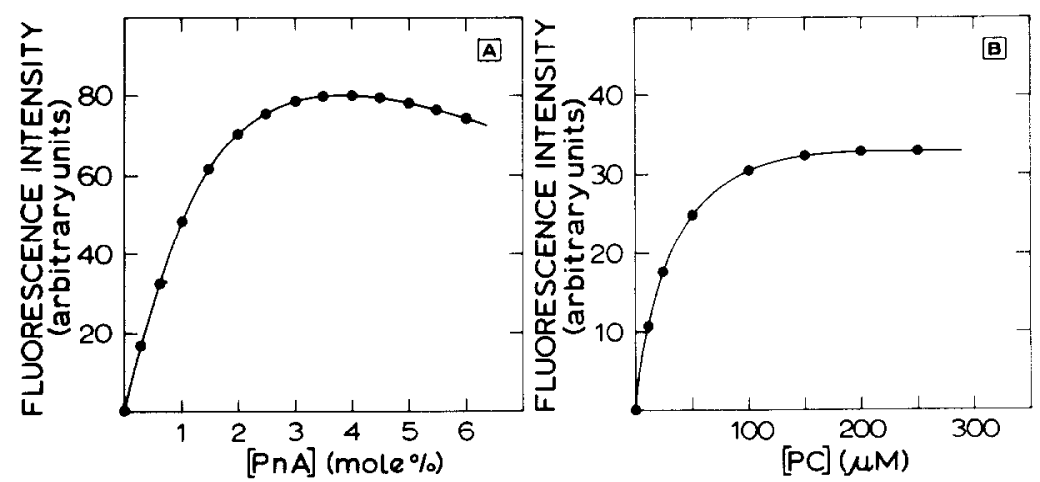

Fig. 2. Fluorescence intensity of cis-parinaric acid in egg PC vesicles as a function of concentration. (A) $2 \mathrm{ml}$ of buffer containing 250 $\mu \mathrm{M}$ of egg PC $/ 5 \mathrm{~mol} \%$ egg phosphatidate vesicles and varying concentrations of cis-parinaric acid. (B) $2 \mathrm{ml}$ of buffer containing 3.8 $\mu \mathrm{M}$ of cis-parinaric acid and various concentrations of egg $\mathrm{PC} / 5 \mathrm{~mol} \%$ egg phosphatidate vesicles. 

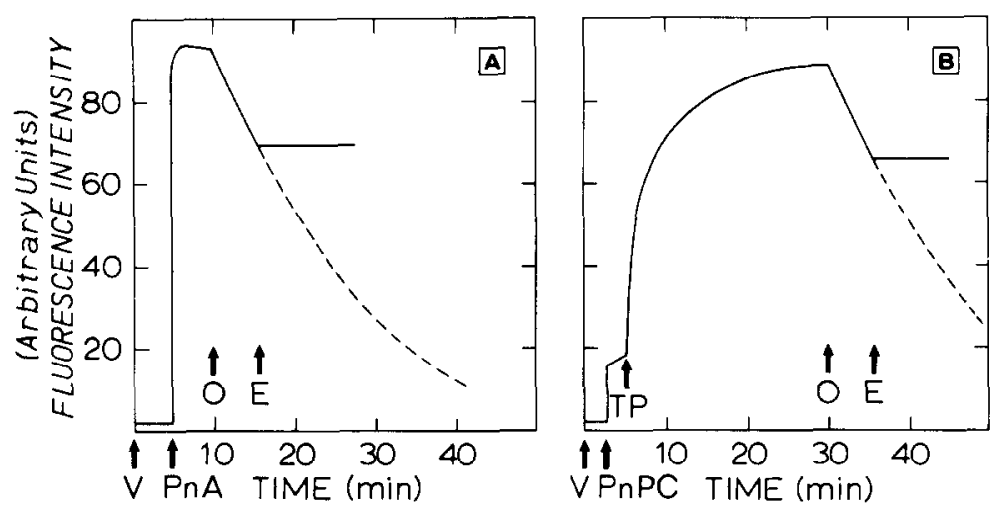

Fig. 3. Incorporation of cis-parinaric acid and parinaroyl PC. into egg PC vesicles and subsequent degradation by $\mathrm{H}_{2} \mathrm{O}_{2}$ - and $\mathrm{Cu}^{2+}$-induced peroxidation. The fluorescence intensity was recorded in time upon incubation of egg PC vesicles with cis-parinaric acid (A) or donor vesicles consisting of parinaroyl PC in the presence of transport protein (B). The incubation was performed at $25^{\circ} \mathrm{C}$ in a total volume of $2 \mathrm{ml}$ of buffer. cis-Parinaric acid and parinaroylPC were added by injection of a small volume of an ethanolic solution. Arrows: V, addition of egg PC vesicles (450 nmol PC); PnA, addition of cis-parinaric acid (3.2 nmol); PnPC, addition of parinaroylPC (4.5 nmol); TP, addition of transfer protein; $\mathrm{O}$, addition of $\mathrm{H}_{2} \mathrm{O}_{2}(40 \mu \mathrm{mol})$ and $\mathrm{CuSO}$ ( $(\mathrm{A}), 0.04 \mu \mathrm{mol}$; (B) $0.26 \mu \mathrm{mol})$; E, addition of EDTA ((A), $0.05 \mu \mathrm{mol}$; (B), $0.30 \mu \mathrm{mol})$.

fluorescence signal. Since such a linear relationship is required in the peroxidation assay, a concentration curve has to be determined for every membrane to be investigated.

As the parinaric acid is partitioned between the water phase and membranes, the concentration of membranes in the water phase will influence the amount of parinaric acid in the membrane and therefore the fluorescence intensity. It is obvious from Fig. 2B that a final concentration of $200 \mu \mathrm{M}$ of lipid avoids any interference of the parinaric acid in the water phase.

In Fig. 3A a typical experiment is shown in which parinaric acid is incorporated in a vesicle membrane. Vesicles were added to buffer, followed by rapid injection of a small volume of an ethanolic solution of parinaric acid. The incorporation of parinaric acid in the membranes is monitored by the increase in fluorescence intensity. Subsequent peroxidative stress applied to the system results in a decrease in fluorescence intensity. In this particular case, a combination of $\mathrm{H}_{2} \mathrm{O}_{2}$ and $\mathrm{Cu}^{2+}$ was used to initiate peroxidation. The peroxidation process can be stopped by adding equimolar amounts of EDTA, which chelates the $\mathrm{Cu}^{2+}$ ions. During the process of peroxidation, the excitation spectrum of parinaric acid changes, as shown in Fig. 1A. Also, the maxima of the ultraviolet absorbance decrease parallel with the fluo- rescence signal, indicating that the polyene structure of the molecule is destroyed by the peroxidation process, rather than that a redistribution of the probe results in a decreased quantum yield. Moreover, after the peroxidation process has been stopped, the fluorescence can be restored by adding fresh parinaric acid up to the original concentration.

Other peroxidation-initiating systems containing phenylhydrazine, cumene hydroperoxide, $t$ butylhydroperoxide with or without transition metal ions were also used and gave comparable results with respect to the loss of the fluorescent properties of parinaric acid, although the kinetics of the different peroxidation reactions varied strongly with the initiators used.

From a comparison of either $\mathrm{H}_{2} \mathrm{O}_{2}$ plus $\mathrm{Cu}^{2+}$ or phenylhydrazine as initiators of peroxidation, it appeared that different peroxidation-initiating systems can induce different ways by which the spectroscopic properties of parinaric acid are destroyed. In both cases, the measured fluorescence signal decreases, the positions of the peaks in the excitation spectrum stay virtually the same, but the relative peak heights arc affected in a different way by the peroxidation process started by these two initiators. The reason for this behaviour is not clear at present and will require further investigation. 
The use of parinaroylPC

When small volumes of an ethanolic solution of parinaroylPC are added to buffer, vesicles of pure parinaroylPC are formed [21]. The fluorescence of the parinaroyl groups is strongly quenched, due to probe-probe interactions (Fig. 3B). In contrast to the free parinaric acid (Fig. 3A), only a slow increase in fluorescence intensity is observed in the presence of other membranes not containing parinaroylPC.

The phosphatidylcholine transfer protein from bovine liver facilitates the exchange of $\mathrm{PC}$ between membranes [22], and it is shown in Fig. 3B that upon addition of this protein an increase in fluorescence intensity occurs. Transfer of probe molecules from the pure parinaroylPC vesicles into the egg $\mathrm{PC}$ vesicles and, in turn, of egg PC molecules into the parinaroylPC vesicles, decreases the probe-probe interactions. hence increasing the observed fluorescence intensity. The dilution of the probe achieved in this way, resulting in an increase in fluorescence intensity, offers the opportunity to monitor the exchange process. Under the experimental conditions of Fig. 3 , the equilibration takes approximately $30 \mathrm{~min}$. Subsequent addition of the peroxidation initiators $\mathrm{Cu}^{2+}$ plus $\mathrm{H}_{2} \mathrm{O}_{2}$ results in a decrease in the fluorescence intensity. This process can be stopped by chelation of the $\mathrm{Cu}^{2+}$ ions with EDTA.

The peroxidation induced changes in the spectroscopic properties of the polyene group in the
PC molecule are virtually identical to those in the polyene group of the free fatty acid and are therefore not shown.

\section{Comparison with other methods}

Fig. 4 shows an experiment in which a comparison of four different measurements of peroxidation is given. The peroxidation conditions chosen lead to a sharp decerase in the concentration of parinaric acid and a loss of linoleic acid, the other polyunsaturated fatty acid present in the vesicle membrane. Also, the formation of both thiobarbituric acid-reactive substances and conjugated dienes, as measured by ultraviolet absorbance, were observed. It is obvious from these results that parinaric acid is much more sensitive towards peroxidative stress than is linoleic acid, an observation that can be expected on basis of the number of double bonds. It is also clear that there exists no direct quantitative relationship between the different methods to measure peroxidation.

Use of the parinaric acid/parinaroylPC method to determine the rate of peroxidation

As fluorescence intensity and probe concentration are linearly related under the assay conditions. the rate of fluorescence decrease of the probe upon application of an oxidative stress can be taken as a reflection of the rate of its peroxidation. Since the peroxidation process can be monitored continuously, measurements of the rate of

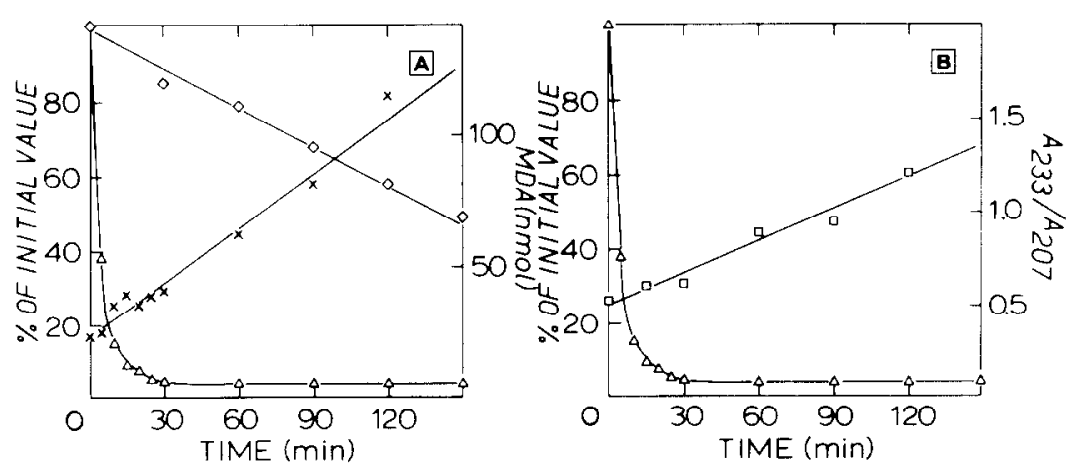

Fig. 4. The time-dependent lipid peroxidation at $37^{\circ} \mathrm{C}$ of vesicles composed of POPC, DLPC and cis-parinaric acid induced by $\mathrm{H}_{2} \mathrm{O}_{2}$ and $\mathrm{Cu}^{2 *}$. At the start of the incubation, the reaction mixture contained $1.7 \mathrm{mM}$ POPC. $1.7 \mathrm{mM}$ DLPC. $35 \mu \mathrm{M}$ cis-parinaric acid. $10 \mathrm{mM} \mathrm{H}_{2} \mathrm{O}_{2}$ and $0.2 \mathrm{mM} \mathrm{CuSO}_{4}$. (A) The reaction as monitored by the relative decrease in fluorescence intensity of cis-parinaric acid $(\Delta)$, the relative decrease in the amount of linoleic acyl chains as determined by GLC $(\diamond)$, and the increase in thiobarbituric acid-reactive substances, expressed as equivalents of malondialdehyde in the sample $(X)$. (B) The relative decrease in fluorescence intensity of $c i s$-parinaric acid $(\Delta)$ and the increase in conjugated dienes $(\square)$, expressed in an oxidation index, $A_{2,3.3} / A_{2 i n}$ 

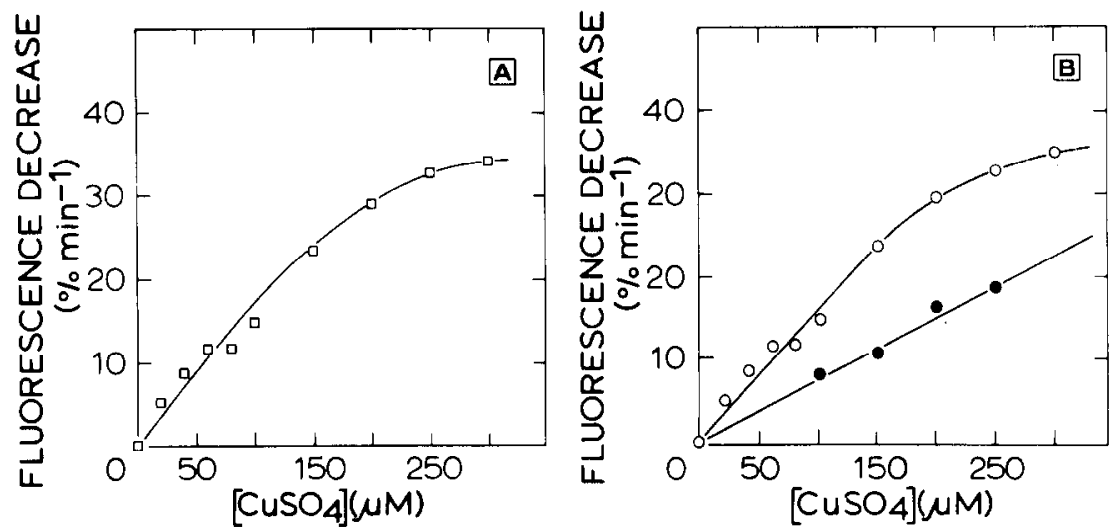

Fig. 5. (A) The rate of $\mathrm{H}_{2} \mathrm{O}_{2} / \mathrm{Cu}^{2+}$-induced peroxidation of cis-parinaric acid in egg $\mathrm{PC} / 5 \mathrm{~mol} \%$ egg phosphatidate vesicles at $25^{\circ} \mathrm{C}$ as a function of $\mathrm{Cu}^{2+}$ concentration. The incubation was performed at $25^{\circ} \mathrm{C}$ in a total volume of $2 \mathrm{ml}$. The incubation mixture contained $225 \mu \mathrm{m}$ egg PC, $1.9 \mu \mathrm{M}$ cis-parinaric acid $(0.8 \mathrm{~mol} \%), 20 \mathrm{mM} \mathrm{H}_{2} \mathrm{O}_{2}$ and $\mathrm{CuSO}_{4}$ as indicated. The rate of probe degradation was taken as the initial rate of fluorescence decrease upon addition of $\mathrm{H}_{2} \mathrm{O}_{2}$ and $\mathrm{CuSO}_{4}$. (B) Comprison of the rates of $\mathrm{H}_{2} \mathrm{O}_{2} / \mathrm{Cu}^{2+}$ induced peroxidation of cis-parinaric acid $(\mathrm{O})$ and parinaroyl PC $(\bullet)$ in egg PC/5 mol\% egg phosphatidate vesicles. Experimental conditions as in (A), parinaroylPC concentration was $1.57 \mu \mathrm{M}(0.7 \mathrm{~mol} \%)$.

peroxidation can be performed in a convenient way. In Fig. 5A, the results of an experiment are shown in which the conditions were virtually identical to those in the experiment shown in Fig. 3A, with respect to the amount of lipid material. The initial rate of fluorescence decrease is determined as a function of the concentration of $\mathrm{Cu}^{2+}$ ions in the solution at constant $\mathrm{H}_{2} \mathrm{O}_{2}$ concentration. The rate of peroxidation is, as expected, strongly de- pendent on the concentration of the initiators. At lower concentrations of $\mathrm{Cu}^{2+}$ a linear relationship seems to exist between the rate of peroxidation and the amount of $\mathrm{Cu}^{2+}$ ions present in the suspension at a given $\mathrm{H}_{2} \mathrm{O}_{2}$ concentration.

In Fig. 5B a comparison is shown of the sensitivity of parinaric acid and parinaroyl PC in egg $P C$ vesicles under virtually identical conditions. It is clear that the free fatty acid is more sensitive to
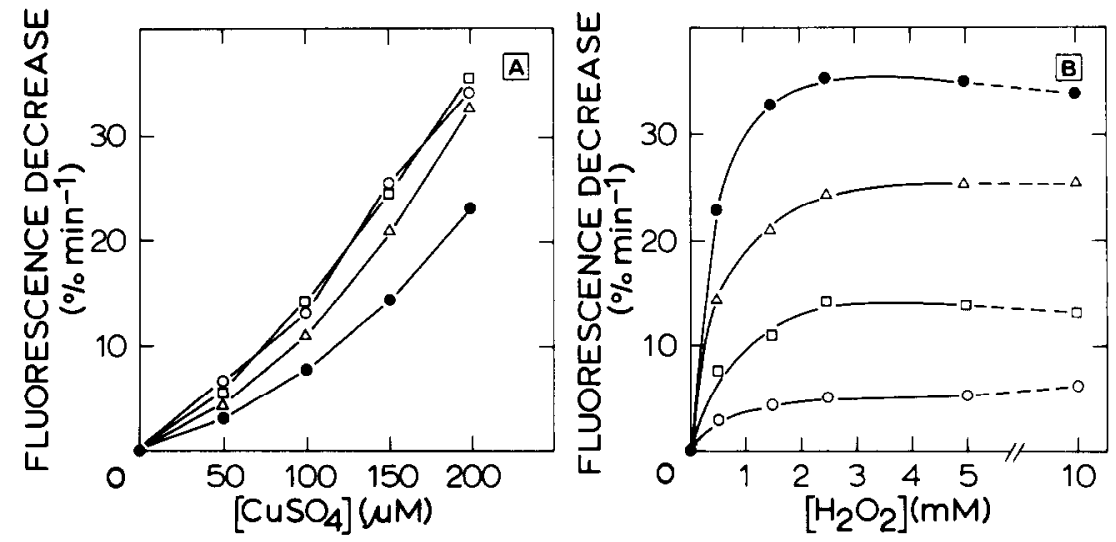

Fig. 6. The relation between probe degradation and initiator concentration at $25^{\circ} \mathrm{C}$. In an egg PC/5 mol\% egg phosphatidate vesicle suspension $(250 \mu \mathrm{M})$, containing $1.87 \mu \mathrm{M}$ cis-parinaric acid $(0.75 \mathrm{~mol} \%)$, peroxidation was initiated by various concentrations of $\mathrm{H}_{2} \mathrm{O}_{2}$ and $\mathrm{Cu}^{2+}$. The rate of probe degradation was taken as the initial rate of fluorescence decrease upon addition of $\mathrm{H}_{2} \mathrm{O}_{2}$ and $\mathrm{CuSO}_{4}$. (A) Results plotted as a function of $\mathrm{Cu}^{2+}$ concentration for $\mathrm{H}_{2} \mathrm{O}_{2}$ concentrations of $0.5 \mathrm{mM}(\bullet), 1.5 \mathrm{mM}(\Delta), 2.5 \mathrm{mM}(\square)$ and $10 \mathrm{mM}(\mathrm{O})$. (B) Results plotted as a function of $\mathrm{H}_{2} \mathrm{O}_{2}$ concentration for $\mathrm{Cu}^{2+}$ concentrations of $50 \mu \mathrm{M}(\mathrm{O}), 100 \mu \mathrm{M}(\square), 150$ $\mu \mathrm{M}(\Delta)$ and $200 \mu \mathrm{M}(\bullet)$. 
the peroxidative stress applied to the system.

The relation between probe degradation and initiator concentration is represented in Fig. 6 for varying concentrations of $\mathrm{H}_{2} \mathrm{O}_{2}$ and $\mathrm{Cu}^{2+}$.

\section{Discussion}

The fluorescent properties of parinaric acid, originating from the conjugated configuration of the four double bonds in the acyl chain, have made this molecule a very useful membrane probe $[6,7]$. The presence of the four double bonds, however, confers the acyl chain of parinaric acid, like other polyunsaturated fatty acids, a high sensitivity towards free radical mediated peroxidation processes. In this study, we show that a peroxidative stress applied to a membrane in which parinaric acid is present in either its free carboxylic form or esterified in PC, results in the loss of the typical properties of the polyenic structure of this molecule.

The decrease in fluorescence intensity that can be observed as a result of this peroxidation process, parallels the peroxidative breakdown of other polyunsaturated fatty acids in the membrane as is shown for linoleic acid in Fig. 4. Also, two other features of peroxidative damage, conjugated diene formation and production of thiobarbituric acid reactive substances, are observed. It seems plausible to assume that degradation of parinaric acid does not proceed by a mechanism exactly identical to that for the other polyunsaturated fatty acids, because the polyenic structure of parinaric acid is basically different. However, the initial stages of peroxidation (i.e., hydrogen abstraction and subsequent double bond shift) will be comparable and it is during the initial stages that the conjugated polyenic structure of parinaric acid is altered sufficiently to result in a complete loss of fluorescent properties at the wavelengths applied.

Since this new method determines directly the breakdown of one particular fatty acid in the membrane, it is not sensitive to all kinds of sideeffects like the widely used thiobarbituric acid test, that does not exclusively measure the production of malondialdehyde. Furthermore, the thiobarbituric acid test provides an evaluation of the amount of peroxidation end-products as a final result of the lipid peroxidation process, whereas the method described here offers a possibility for one-line monitoring of the peroxidation process in its initial stages. Our method can be compared to the quantitative determination of fatty acids by gas chromatography.

\section{The use of a probe molecule}

As extensively described in the literature [4], only a qualitative relationship exists between the different assay systems used to monitor peroxidation. The formation and detection of the various peroxidation products are found to lack a direct quantitative relationship with the decrease in polyunsaturated fatty acids. Also, the various fatty acyl chains differ greatly in their sensitivity towards peroxidative stress [23]. The fluorescent octadecatetraenoic acid (parinaric acid) that we used in this study is very sensitive to peroxidation and. although we made no direct comparison with all the other naturally occurring polyunsaturated fatty acids, the assumption seems justified that it has a higher sensitivity towards peroxidation than most of the others. Therefore, the breakdown of this fatty acid does not necessarily reflect the extent of peroxidative damage inflicted upon a membrane. This depends on the composition of the membrane and only a quantitative characterization of all fatty acyl species can give such direct information. Nevertheless, the degradation of this probe, as a polyunsaturated fatty acid sensitive to peroxidation, will give direct information on the sensitivity towards a peroxidative stress of the membrane in which it is incorporated.

\section{Parinaric acid or parinaroylPC}

From our results it is clear that parinaric acid, in its free carboxylic form as well as esterified in PC, is sensitive to peroxidation. The free acid, however, is more sensitive to this process, as can be seen from Fig. 5B. Both probes, especially at the concentrations used, are thought not to disturb the membrane to any appreciable extent. The exact location, however, of a free fatty acid in a membrane is not known and may also depend on the physical state of the membrane. The parinaro$\mathrm{ylPC}$, on the other hand, is likely to behave as any other PC molecule in the membrane. These probably different locations in the membrane may explain the difference in susceptibility of the free 
acid and the acid esterified in PC.

It seems reasonable to assume that the parinaroylPC will give more exact information regarding the peroxidation susceptibility of the polyunsaturated fatty acids present in the membrane phospholipids. On the other hand, the indispensable use of a transfer protein to introduce the parinaroylPC in a biological membrane will make its application more limited than that of the free acid.

\section{Sensitivity, ease of use}

A great advantage of the parinaric acid/ parinaroylPC method is the fact that peroxidation can be measured directly and continuously in a membrane during an experiment, without the need of sampling or derivatization afterwards. This makes the method suitable for time-scale experiments and very useful for kinetic studies. Furthermore, it is relatively simple and quick to perform.

Its sensitivity is superior to that of several other methods. A decrease in parinaric acid concentration of $5 \%$ per minute, from an original amount of $2 \mathrm{nmol}$ in $400 \mathrm{nmol}$ of membrane lipid material, can be easily measured. This means that a decrease of $0.1 \mathrm{nmol}$ per min can be monitored. Depending on the volumes and fluorimeter used, the above amounts may even be scaled down.

\section{Application, interferences}

In principle, the method can be applied to various kinds of membrane, as long as the amounts of lipid material are in the range as described. Like the limitation in gas chromatographic analysis, it is essential to keep in mind that in metabolically active cells the level of polyunsaturated fatty acid may be subject to considerable changes in time, which may also affect the probe molecules.

Also, changes in the quantum yield as a result of changes in distribution of the probe or changes in physical properties of the membrane, might interfere with the determination. On the other hand, the extent of this kind of interference depends very strongly on the conditions chosen and the experimental timescale.

The presence of highly fluorescent or absorbing materials in the wavelength region used for the assay can make this method less sensitive or even inapplicable. As an example, the method could only be used on pink or white ghosts and not on intact red blood cells, due to the strong absorbance by the hemoglobin present at high concentrations.

\section{Conclusion}

The new fluorimetric assay presented here offers the possibility to monitor peroxidation processes in a membrane, especially during the initial stages.

This new method can give information on the susceptibility of a membrane to free radical mediated peroxidation processes in a highly sensitive way. Effects that changes in the lipid core or the protein constituents of a model or biological membrane might have on this susceptibility can easily be detected.

The method can be used to measure the kinetics of different peroxidation initiators, as well as the effectiveness of peroxidation inhibiting systems present, such as vitamin $E$, catalase and superoxide dismutase.

\section{Acknowledgmeents}

Mrs. M. van Linde-Sibenius Trip is gratefully acknowledged for the purification of the transfer protein. This investigation was carried out under the auspices of The Netherlands Foundation for Chemical Research (S.O.N.) and with financial aid from The Netherlands Organization for the Advancement of Pure Research (Z.W.O.). This work was in part supported by National Institutes of Health Grant AM 32094, Grant HL 37593 and NATO Travel Grant 85/0665.

\section{References}

1 Freeman, B.A. and Crapo, J.D. (1982) Lab. Invest. 47, 412-426

2 Mead, J.F. (1976) in Free Radicals in Biology, Vol. I (Pryor, W.A., ed.), pp. 51-68, Academic Press, New York

3 Frankel, E.N. (1985) Prog. Lipid Res. 23, 197-221

4 Slater, T.F. (1984) Methods Enzymol. 105, 283-293

5 Sklar, L.A., Hudson, B.S, and Simoni, R.D. (1975) Proc. Natl. Acad. Sci. USA 72, 1649-1653

6 Sklar, L.A., Hudson, B.S., Petersen, M. and Diamond, J. (1977) Biochemistry 16, 813-819

7 Sklar, L.A., Hudson, B.S. and Simoni, R.D. (1977) Biochemistry $16,819-828$

8 Pugh, E.L., Kates, M. and Szabo, A.G. (1982) Chem. Phys. Lipids 30, 55-69 
9 Pjura, W.J., Kleinfeld, A.M. and Karnovsky, M.J. (1984) Biochemistry 23, 2039-2043

10 Christiansson, A., Kuypers, F.A., Roelofsen, B., Wirtz, K.W.A. and Op den Kamp, J.A.F. (1984) Chem. Phys. Lipids 35, 247-258

11 Kuypers, F.A., Roelofsen, B., Berendsen, W., Op den Kamp, J.A.F. and Van Deenen, L.L.M. (1984) J. Cell Biol. 99, 2260-2267

12 Dodge, J.T. and Phillips, G.B. (1967) J. Lipid Res. 8, $667-675$

13 Somerharju, P., Brockerhoff, H. and Wirtz, K.W.A. (1981) Biochim. Biophys. Acta 649, 521-528

14 Westerman, J., Kamp, H.H. and Wirtz, K.W.A. (1983) Methods Enzymol. 98, 581-586

15 Rose, H.G. and Oklander, M. (1965) J. Lipid Res. 6, 428-431
16 Rouser, G.. Fleischer. S. and Yamamoto, A. (1970) Lipids 5, 494-496

17 Morrison, W.R. and Smith, I.M. (1964) J. Lipid Res. 5 , 600-608

18 Klein, R.A. (1970) Biochim. Biophys. Acta 210, 486-489

19 Bidlack, W.R. and Tappel, A.L. (1973) Lipids 8, 177-182

20 Placer, Z.A., Cushman, L.L. and Johnson, B.C. (1966) Anal. Biochem. 16, 359-364

21 Batzri. S. and Korn, E.D. (1973) Biochim. Biophys. Acta 298. 1015-1019

22 Demel, R.A., Wirtz, K.W.A., Kamp, H.H., Geurts van Kessel. W.S.M. and Van Deenen. L.L.M. (1973) Nature New Biol. 246, 102-105

23 Mowri, H., Nojima, S. and Inoue, K. (1984) J. Biochem. 95. $551-558$ 